\title{
Les nombreux visages de l'élargissement idiopathique aigu de la tache aveugle.
}

\author{
Amiee Ho, DO \\ Professeur adjoint, \\ Collège d'optométrie de \\ l’Université du Pacifique \\ Denise Goodwin, DO, FAAO \\ Professeur, \\ Collège d'optométrie de \\ l’Université du Pacifique
}

\begin{abstract}
Résumé
Le présent article présente une recension des écrits et une série de cas rétrospective d'élargissement idiopathique aigu de la tache aveugle chez deux femmes de race blanche et un homme hispanique. Les constatations les plus caractéristiques comprennent une photopsie unilatérale, un élargissement de la tache aveugle, la perte des zones ellipsoïde et d'interdigitation en tomographie par cohérence optique (TCO) et un ERGmf anormal. Il s'agit d'une maladie de la rétine externe qui partage des traits communs avec la rétinopathie occulte externe zonale aiguë (AZOOR), ce qui peut fausser le diagnostic. L'imagerie en autofluorescence peut aider à différencier les conditions. La diffusion d'un plus grand nombre de rapports de cas décrivant différentes présentations de ce trouble de la rétine externe rare devrait aider à améliorer la compréhension clinique de ces affections.
\end{abstract}

\section{MOTS CLÉS :}

Élargissement idiopathique aigu de la tache aveugle; dysfonction idiopathique de la rétine externe associée à des anomalies du champ visuel ; nerf optique; maladie de la rétine externe; photopsie; champ visuel

\section{INTRODUCTION}

L'élargissement idiopathique aigu de la tache aveugle (ÉIATA), décrite pour la première fois en $1988^{1,}$ est une maladie rare qui affecte la rétine péripapillaire. La maladie se traduit par l'apparition soudaine d'un scotome contigu à la tache aveugle physiologique malgré l'absence d'un œdème papillaire correspondant. La taille du scotome varie, mais il s'agit généralement d'un déficit absolu avec des marges abruptes qui ne progresse pas au fil du temps. L'examen du fond d'œil est souvent normal ou seulement légèrement altéré et, par conséquent, l'état est souvent mal diagnostiqué ${ }^{2-4}$. Il est couramment diagnostiqué à tort comme une névrite optique en raison de la démographie et de la perte de vision malgré une santé oculaire relativement normale, ou comme migraine avec aura en raison de la photopsie.

L'étiologie de l'ÉIATA est inconnue. De multiples mécanismes ont été proposés pour les maladies de la rétine externe, y compris une étiologie infectieuse ou auto-immune ${ }^{5}$. Les facteurs hormonaux ou génétiques peuvent jouer un rôle ${ }^{4}$. Les patients sont généralement des femmes âgées de 19 à 53 ans $^{4}$, bien que des cas aient été signalés chez les hommes ${ }^{1,3,6}$ L'origine ethnique n'est souvent pas mentionnée dans les études de cas disponibles ${ }^{4,7}$, mais l'ÉIATA a été signalée chez les caucasiens8 et les asiatiques ${ }^{3,6}$.

Dans la plus grande série à ce jour, Volpe et coll ${ }^{4}$. ont décrit les cas de 27 patients atteints d'ÉIATA recueillis sur une période de huit ans. Le symptôme le plus courant chez les patients atteints d'ÉIATA est la baisse de vision (92\%), classée comme étant une vision floue, des parties manquantes du champ de vision, des taches ou la sensation de 
regarder à travers une pellicule 4 . Les autres symptômes comprennent un phénomène visuel positif (85 \%), comme la photopsie, l'effet de "kaléidoscope ", un mouvement à l'intérieur du scotome, des lumières colorées, ou un phénomène similaire à l'image rémanente produite après avoir regardé une ampoule électrique. L'acuité visuelle est normale chez $60 \%$ des patients et, si elle est anormale, se situe entre 20/25 et 20/50 chez presque tous les patients ${ }^{4}$. Les autres résultats cliniques comprennent un léger œè̀me papillaire (44\%), une dyschromatopsie (33\%), un déficit pupillaire afférent (30\%), des changements des pigments péripapillaires (22\%), des taches blanches rétiniennes (18\%) et une décoloration péripapillaire sous-rétinienne grisâtre $(15 \%)^{4}$. Au fil du temps, une cicatrisation choriorétinienne qui correspond au déficit du champ visuel est susceptible de se développer'.

La tomographie par cohérence optique (TCO) montrera la perte de la zone ellipsoïde et de la ligne d'interdigitation dans la zone péripapillaire concernée. L'électrorétinogramme multifocal (ERGmf) est essentiel au diagnostic de l'ÉIATA. Les résultats d'un électrorétinogramme plein champ sont souvent normaux ${ }^{4}$ parce qu'il enregistre une moyenne de l'activité rétinienne globale et que la rétine saine masque les lectures anormales. Un électrorétinogramme focal ou multifocal révélera des anomalies localisées qui correspondent à la perte du champ visuel. Les anomalies observées sur l'ERGmf persisteront après la résolution d'autres signes, et il peut y avoir des anomalies bilatérales malgré une présentation autrement unilatérale?.

Les diagnostics différentiels qui entraînent un élargissement de la tache aveugle comprennent l'œdème de la tête du nerf optique (p. ex., papilledema) qui cause le déplacement antérieur de la rétine péripapillaire. Dans ces cas, la taille de la tache aveugle correspond à une anomalie visible. La nébulite optique démyélinisante, qui est la plus courante chez les jeunes femmes, est une cause atypique d'élargissement de la tache aveugle. La santé oculaire est normale dans les deux tiers de ces cas en raison de l'implication rétrobulbaire. Il y a généralement de la douleur associée aux mouvements oculaires, un déficit pupillaire afférent important et des anomalies de vision des couleurs. La TCO peut aider à distinguer la névrite optique démyélinisante de l'ÉIATA, car la névrite optique implique la rétine interne (cellules ganglionnaires de la rétine et couche de fibres nerveuses) et ne devrait pas causer de perturbation des couches rétiniennes externes. Avec la névrite optique démyélinisante, les résultats de l'ERGmf seront normaux, puisque le test mesure l'activité de la rétine extérieure. D'autres diagnostics différentiels comprennent les rétinopathies auto-immunes, comme la rétinopathie associée au cancer ou la rétinopathie associée au mélanome, dans lesquelles le fond de l'œil semble normal dans les premiers cas. L'apparition de ces affections sera plus lente que celle de l'ÉIATA et progressera sans intervention5. De plus, une myopie élevée peut causer une tache aveugle légèrement agrandie ainsi que des anomalies péripapillaires visibles et des symptômes de photopsie.

Un élargissement de la tache aveugle attribuable à une maladie de la rétine externe peut être isolé, comme dans le cas de l'ÉIATA, ou associé à d'autres conditions, y compris le syndrome des taches blanches multiples évanescentes (MEWDS), la neurorétinopathie maculaire aiguë, la choroïdite multifocale, la choroïdopathie ponctuée interne (PIC), la rétinopathie externe annulaire aiguë et la rétinopathie occulte externe zonale aiguë (AZOOR). Le chevauchement de ces affections, qui touchent toutes la rétine externe et se produisent principalement chez les femmes jeunes ou d'âge moyen, a amené de nombreuses personnes à se demander si ces affections ne seraient pas toutes des variations d'une seule maladie connue sous le nom d'AZOOR complexe $\mathrm{e}^{3-5}$. L'amélioration des appareils d'imagerie et l'attention portée aux différences mineures peuvent aider à faire la distinction entre les affections.

Il n'existe aucun traitement qui a fait ses preuves pour l'ÉIATA. La photopsie devrait diminuer en 3 à 4 mois. La perte du champ visuel peut régresser légèrement, mais elle ne se résorbe généralement pas complètement, bien que certains rapports indiquent le contraire ${ }^{4,9,10}$. Le premier rapport décrivant l'ÉIATA a signalé la résolution des symptômes de la maladie dans les 2 à 3 mois et une tache aveugle normale chez 3 des 7 patientsl. Il a été avancé que certains de ces patients pouvaient avoir représenté des cas tardifs de MEWDS dans lesquels les lésions du fond d'œil avaient été résolues ${ }^{8}$. Volpe et coll. ${ }^{4}$ ont suivi 10 des 27 patients de leur série de cas : ils présentaient tous une diminution de la photopsie, mais aucune amélioration de la tache aveugle n'a été observée chez aucun des 10 patients. Les anomalies révélées par ERGmf peuvent persister même si le déficit du champ visuel disparait, et peuvent permettre de détecter une dysfonction rétinienne subclinique ${ }^{8}$. Une récidive est possible, quoiqu'il s'agisse d'un événement rare. 
Nous décrivons ici trois présentations d'ÉIATA différentes qui s'ajoutent aux rapports de cas existants. Le premier cas est une présentation classique de la maladie. Le deuxième cas est, à notre connaissance, le premier cas signalé chez un homme hispanique. Le troisième cas démontre la nature intermittente potentielle de la maladie et n'a pas encore été signalé. Ces exemples de cette maladie rare peuvent aider à mieux définir et classer le diagnostic de l'ÉIATA.

\section{RAPPORT DE CAS}

Cas 1 : Une femme de 28 ans de race blanche a été aiguillée pour exclure la névrite optique démyélinisante. Elle a remarqué une vision trouble constante d'apparition soudaine, et des éclairs lumineux du côté temporal du champ de vision de l'œil gauche pendant deux semaines. Elle n'avait pas eu de fièvre ou de maladie récemment, elle ne prenait pas de médicaments, et elle a déclaré que les mouvements oculaires ne causaient aucune douleur. L'acuité visuelle était de 20/20 dans l'œil droit et 20/20-1 dans l'œil gauche. Il y avait un léger déficit pupillaire afférent dans l'œil gauche. Les planches pseudo-chromatiques ont révélé un léger déficit rouge-vert et une désaturation rouge de $20 \%$ dans l'œil gauche. Le fond de l'œil gauche avait des marges de disque légèrement indistinctes avec un croissant scléral et chorö̈dien (fig. 1A). Une TCO de la tête du nerf optique a montré une légère augmentation de l'épaisseur de la couche de fibre nerveuse rétinienne péripapillaire inférieure dans l'œil gauche, et une TCO de la macula a montré une perte de la couche nucléaire externe, de la membrane limitante externe, de la zone ellipsoïdale et de la zone d'interdigitation s'étendant sur un tiers de la distance entre la marge temporale du disque optique et la fovéa (fig. 1B). L'analyse du champ visuel (figure 1C) a révélé la présence d'un scotome temporal profond entourant la tache aveugle dans l'œil gauche. L'imagerie par résonance magnétique (IRM) avec contraste n'a montré aucun signe de névrite optique ou de démyélinisation. L’ERGmf (fig. 1D) a montré une diminution significative de l'amplitude et du temps implicite dans la zone correspondant à la perte du champ visuel. Huit semaines plus tard, la photopsie avait disparu, mais le déficit du champ visuel et la perte rétinienne externe observée par TCO étaient stables. Le déficit pupillaire afférent à gauche était toujours présent.

Cas 2 : Un homme hispanique de 24 ans avait remarqué une tache noire circulaire constante dans le coin supérieur gauche de sa vision neuf jours plus tôt. Quand il fermait les yeux, la tache apparaissait comme une lumière blanc verdâtre. Il n'avait pas eu de maladie récente ni de symptômes ressemblant à ceux de la grippe. Les antécédents oculaires et médicaux n'ont rien révélé de marquant. Il ne prenait pas de médicaments. La MAVC était de 20/20 OD et 20/15 OS. Il n'y avait aucun déficit pupillaire afférent et la vision des couleurs était normale. L'examen du segment postérieur a révélé la présence d'un disque allongé horizontalement non incliné avec un croissant scléral inférieur dans les deux yeux (fig. 2A). La TCO de la macula a montré une perte de la membrane limitante externe, de la zone ellipsoïde et de la zone d'interdigitation en temporal du disque optique (fig. 2B) dans l'œil gauche. L'analyse du champ visuel (figure $2 \mathrm{C}$ ) a révélé la présence d'un scotome temporal profond entourant la tache aveugle dans l'œil gauche. L'ERGmf (fig. 2D) a montré une diminution significative de l'amplitude et une diminution légère du temps implicite dans la zone correspondant à la perte du champ visuel. Six semaines plus tard, il remarquait moins la tache quand les deux yeux étaient ouverts. Le champ visuel et l'ERGmf étaient semblables aux lectures précédentes. Deux ans après l'événement initial, le patient a déclaré que la tache était toujours présente, mais qu'elle n'était perceptible que s'il fermait l'œil droit. Bien que certaines différences puissent être attribuées à l'utilisation de stratégies d'essai différentes, un petit scotome contigu à la tache aveugle de l'œil gauche est demeuré (figure 2E). L'œil gauche montrait une hypoautofluorescence importante dans la région péripapillaire sans hyperautofluorescence importante (fig. 2F). La zone de diminution de l'amplitude révélée par l'ERGmf était plus petite (fig. 2G). Aucun changement évident n'a été constaté par TCO.

Cas 3 : Une femme de 24 ans de race blanche a remarqué une zone scintillante dans le champ temporal de l'œil droit perceptible de façon intermittente, pendant trois ans. Le scintillement durait environ 15 secondes et était suivi par une tache aveugle dans la même zone qui devait durer quelques heures. Son état de santé n'avait rien de marquant, et elle prenait du lévonorgestrel/éthinylestradiol. Les MAVC étaient de 20/15 pour chaque œil. Il n'y avait aucun déficit pupillaire afférent et la vision des couleurs était normale. Avant et après un épisode, il y avait un halo de 360 degrés autour du nerf optique droit (fig. 3A). Au cours d'un épisode, le halo péripapillaire s'est agrandi (fig. 3B) et la tache aveugle a été légèrement élargie à l'œil droit (fig. 3C). Avant et après l'épisode, les champs visuels n'ont rien révélé de marquant. Entre les épisodes, une trame de 5 lignes a montré une petite zone de perturbation des zones d'ellipsoïde et d'interdigitation en temporal du nerf optique de l'œil droit, mais la zone correspondante de l'œil gauche n’a rien révélé de marquant (fig. 3D). L'ERGmf réalisé entre les épisodes a montré une amplitude et un temps implicite normaux. Toutefois, on a observé une diminution et un léger retard de la réponse de la rétine en nasal de l'œil droit par rapport au reste des réponses de cet œil (fig. 4E). 
Figure 1 : Une femme de 28 ans de race blanche atteinte d'ÉIATA dans l'œil gauche présente des marges de disque légèrement indistinctes $(A)$, une perte des couches rétiniennes externes adjacentes à la tête du nerfoptique par TCO de la macula (B), un élargissement de la tache aveugle $(C)$, et une réduction significative de l'amplitude et du temps implicite dans la zone concernée sur l'ERGmf (D). Aucune des analyses de l'œil droit non atteint n'a révélé quelque chose de particulier.

A.
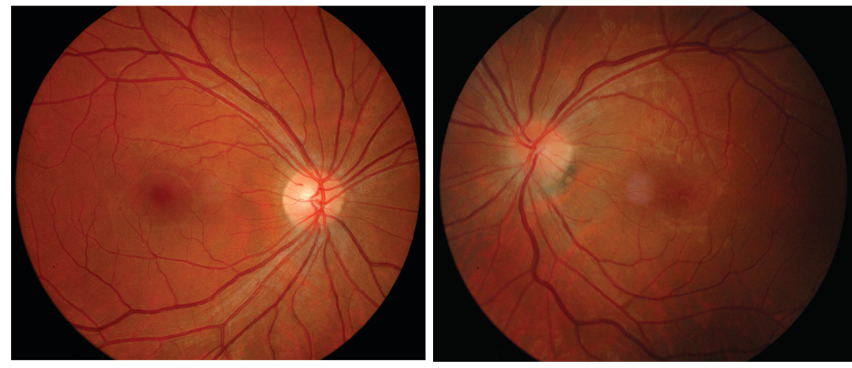

Fixation Losses: $0 / 22$

C. Fest Duration: $09: 03$

Fovea: $31 \mathrm{~dB}$ -

Strategy: SITA-Standarc

$R X: D S \quad D C X$

Age: 28

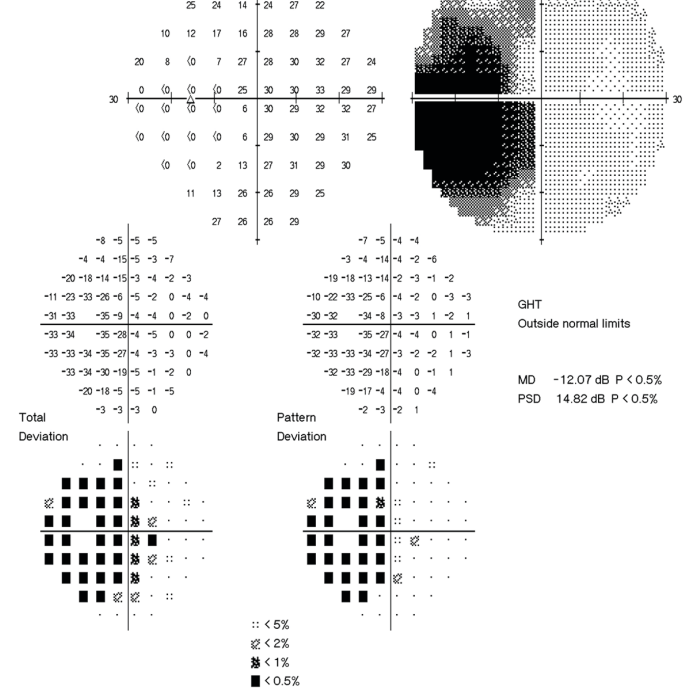

B.

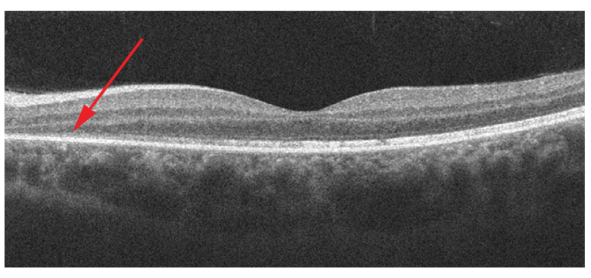

D.

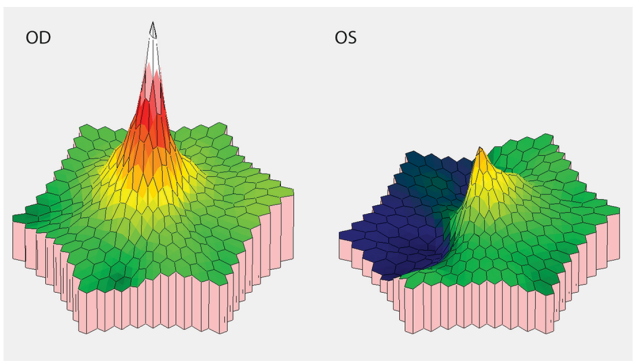


Figure 2 : Un homme hispanique de 24 ans atteint d'ÉIATA dans l'œil gauche présente des nerfs optiques allongés horizontalement avec un croissant scléral inférieur dans les deux yeux $(A)$, une perte des couches rétiniennes externes par TCO de la macula (B), un élargissement de la tache aveugle (C), et une réduction significative de l'amplitude et du temps implicite dans la zone concernée sur l'ERGmf(D) uniquement dans l'œil gauche. Deux ans plus tard, un petit scotome persiste dans l'œil gauche (E). L'autofluorescence du fond d'œil (F) montre une hypoautofluorescence dans la zone du croissant scléral dans les deux yeux avec un léger croissant hyperautofluorescent en nasal dans l'œil droit, et une plus petite zone d'amplitude réduite reste dans l'œil gauche sur l'ERGmf $(G)$.

A.

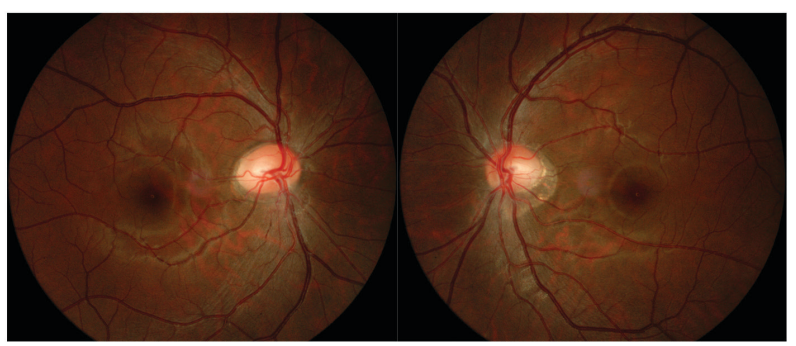

B.

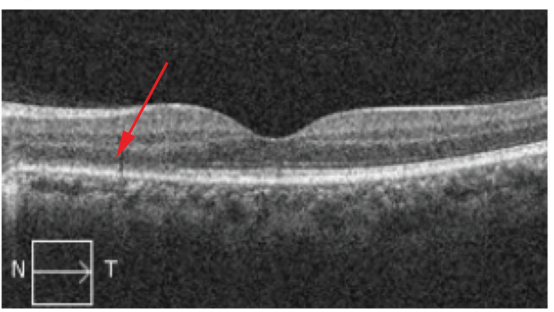

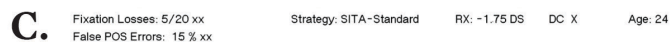
False POS Errors: $15 \% \times x$
False NEG Errors: $6 \%$ False NEG Errors: 6
Test Duration: $09: 12$ Fovea: $38 \mathrm{~dB}$

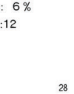

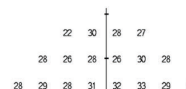

$\begin{array}{lllllllll}28 & 28 & 28 & 31 & 32 & 33 & 20 & 25\end{array}$

$\begin{array}{llllllllll}31 & 25 & 28 & 23 & 28 & 35 & 34 & 31 & 26 & 28\end{array}$

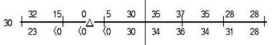

$\begin{array}{llllllllll}26 & 27 & 11 & 16 & 32 & 30 & 30 & 34 & 3 & 31\end{array}$

$\begin{array}{llllllll}2 & 30 & 2 & 33 & 32 & 33 & 2\end{array}$

$\begin{array}{llllll}32 & 32 & -32 & 30 & 35\end{array}$

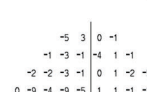

$\begin{array}{llll}33 & 31 & 20 & 20\end{array}$

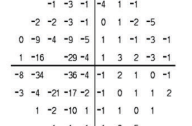

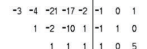

Total
Deviation

$29 \quad-62=-1-2$

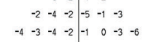

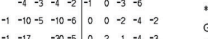

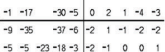

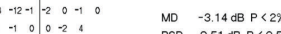

Pattern
Deviation

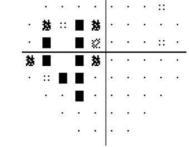

$<5 \%$
$<2 \%$
$<1 \%$
$<0.5 \%$

E.
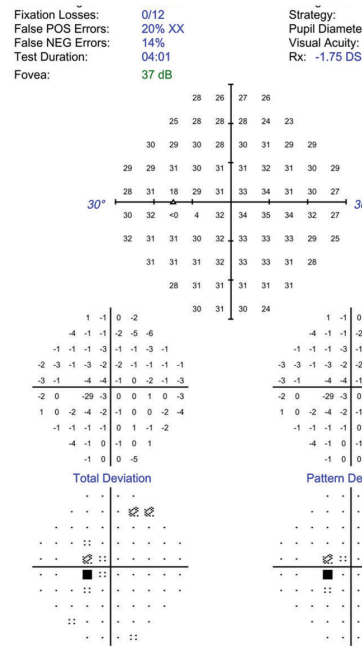

SITA-Fast
$8.5 \mathrm{~mm}^{\circ}$
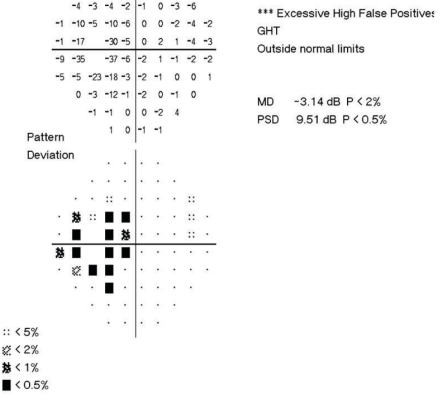$$
{ }_{26}^{26}
$$$$
\begin{array}{llll}
30 & 31 & 29 & 29 \\
32 & 31 & 30
\end{array}
$$
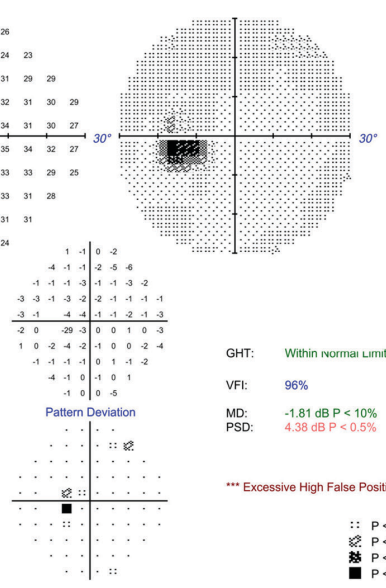

GHT:

VFl: $\quad 96 \%$

$\begin{array}{ll}\text { MD: } & -1.81 \mathrm{~dB} P<10 \% \\ \text { PSD: } & 4.38 \mathrm{~dB} P<0.5 \%\end{array}$

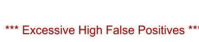

D.

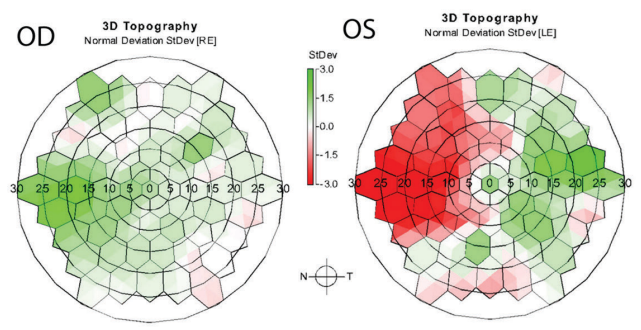

F.
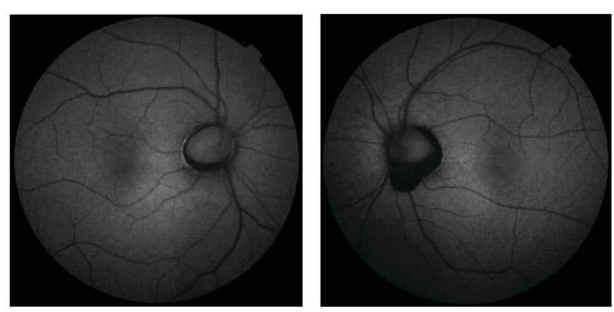

G.

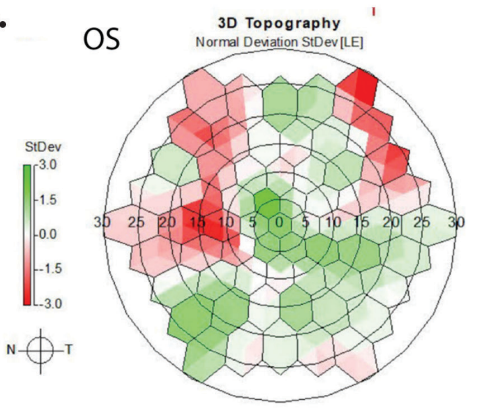


Figure 3 : Femme de 24 ans de race blanche atteinte d'un ÉIATA intermittent à l'œil droit. Les photos du fond d'œil avant (A) et pendant (B) un épisode montrent une augmentation du halo gris entourant la tête du nerf optique pendant l'épisode. Il y a un élargissement de la tache aveugle dans l'oil droit pendant un épisode (C). Il y a une petite zone de perturbation des couches rétiniennes externes en temporal du nerf optique de l'œil droit $(D)$ et des réponses relativement réduites et légèrement retardées dans la rétine de l'œil droit en nasal sur l'ERGmf (E).

A.

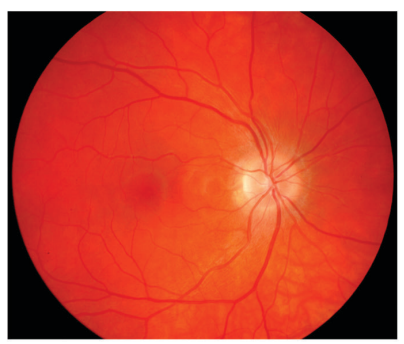

B.

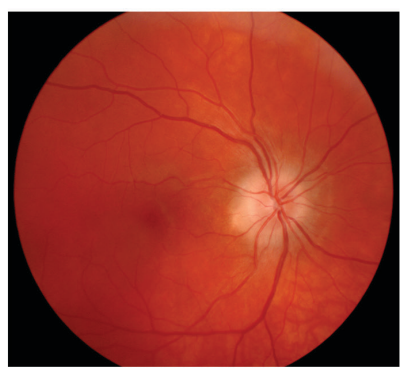

C.

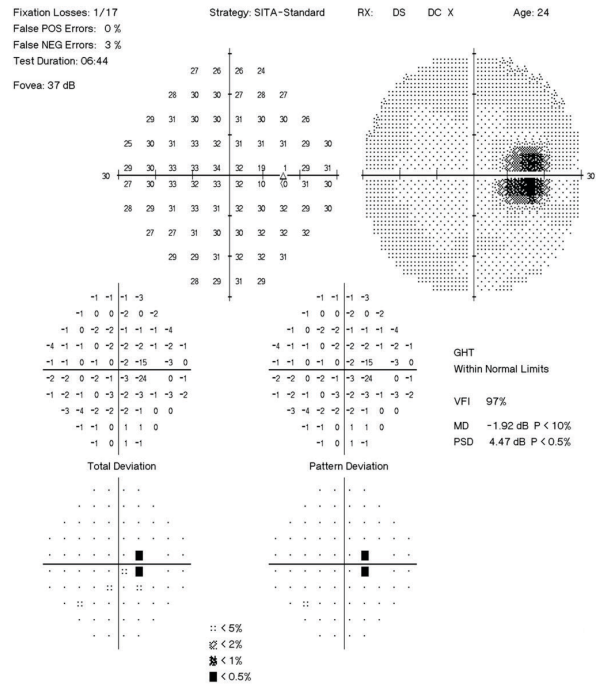

D.
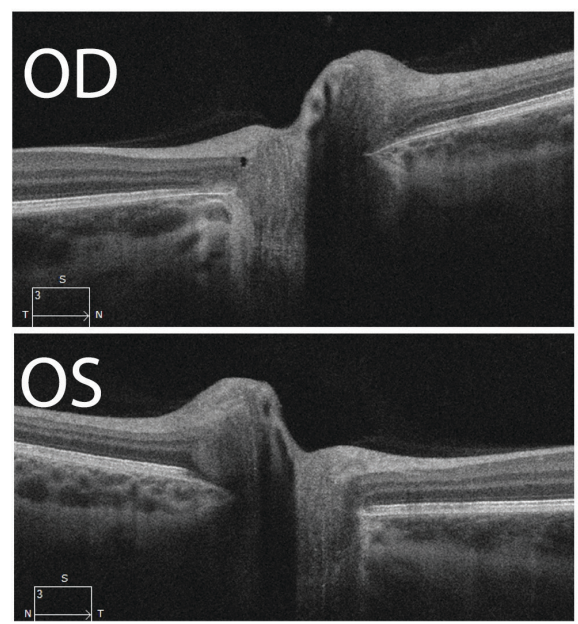

E.
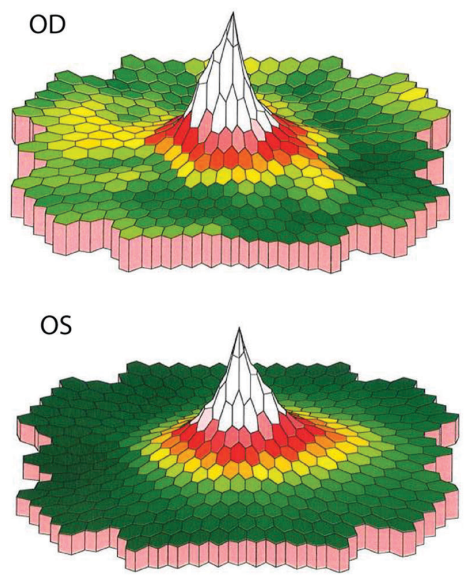
Figure 4 : Multiples taches blanches éparses dans l'œil droit (A) associées au MEWDS. Présence d'une granularité de pigment maculaire dans l'oil droit après la résolution des taches blanches (B). La TCO de la macula (C) montre l'atteinte de la zone ellipsoïde et de la zone d'interdigitation à la fovéa de l'œil droit. L'ERGmf(D) montre une zone d'amplitude réduite qui s'étend en nasal depuis la fovéa de l'œil droit.

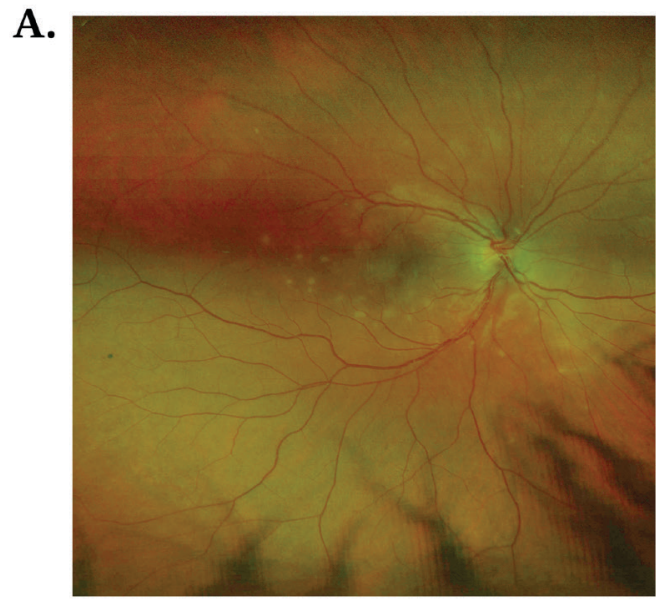

C.

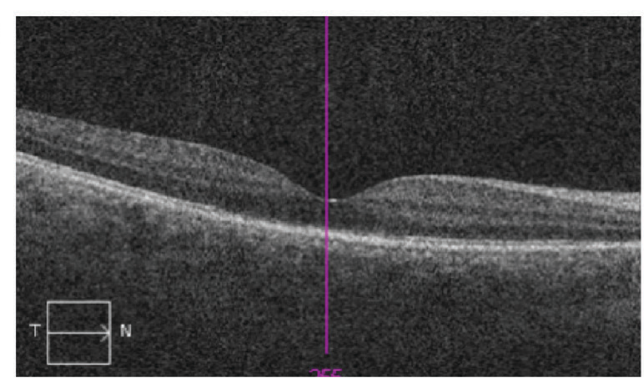

B.

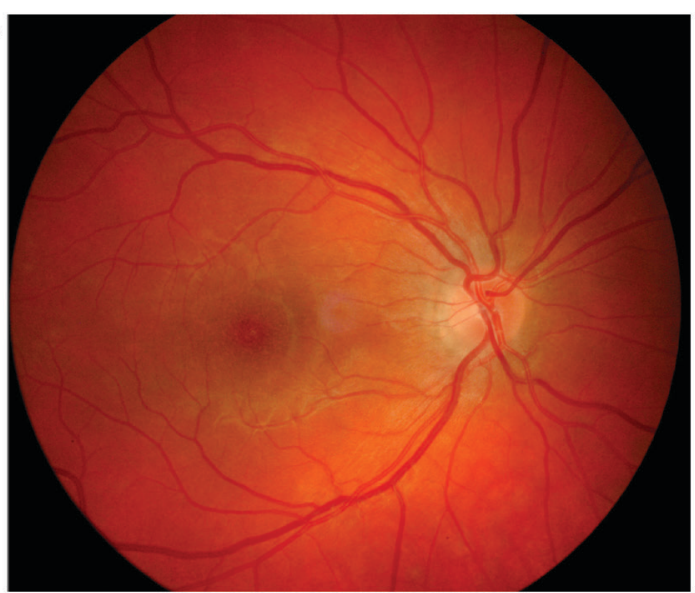

D.

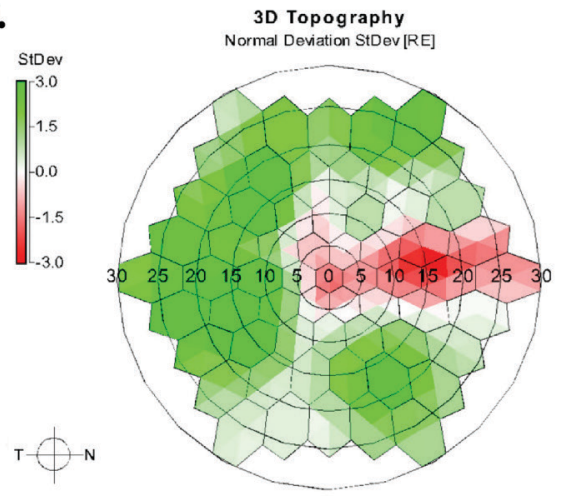

\section{DISCUSSION}

L'élargissement idiopathique aiguë de la tache aveugle (ÉIATA) est une maladie rare. Dans le présent article, nous ajoutons trois cas d'ÉIATA à la documentation existante. Le premier cas est représentatif d'une présentation classique de l'ÉIATA, bien qu'il confirme encore une fois que la maladie continue d'être mal diagnostiquée, car elle a été diagnostiquée au départ comme une névrite optique démyélinisante. Aucun diagnostic n'avait été posé au moment de l'aiguillage pour les deuxième et troisième cas. Le deuxième cas est inhabituel en ce sens qu'il est apparu chez un homme hispanique, alors que la plupart des cas signalés d'ÉIATA se produisent chez les jeunes femmes de race blanche. À notre connaissance, il s'agit du premier cas signalé d'ÉIATA chez un patient hispanique de sexe masculin. Enfin, en ce qui concerne le troisième cas, nous ajoutons une présentation intermittente inhabituelle d'ÉIATA.

Comme on l'a vu dans ces trois cas d'ÉIATA, le disque optique a souvent un aspect anormal, comme un disque congestionné ou un léger œdème discal ${ }^{9}$. Deux des trois patients (cas 1 et 3 ) présentaient un léger œedème discal et un halo péripapillaire grisâtre. Fait intéressant, la zone grisâtre s'est considérablement agrandie pendant les événements intermittents du cas 3. Deux des cas montraient un nerf allongé horizontalement avec une atrophie péripapillaire importante. Dans les trois cas, les changements péripapillaires observés dans l'œil en cause étaient plus importants que ceux de l'œil sain. 
Conformément à la rétinopathie externe, les cas que nous avons présentés ont montré un ERGmf anormal correspondant à la perte du champ visuel. Tous les cas ont montré une diminution de l'amplitude et du temps implicite. Fait intéressant, pour le cas 3, qui présentait un ÉIATA intermittent, l'ERGmf a montré des anomalies entre les épisodes, même si la patiente n'avait aucun symptôme ni perte de champ visuel. En plus des anomalies révélées par l'ERGmf, l'autre caractéristique diagnostique déterminante de l'ÉIATA, soit les anomalies de la rétine externe, y compris la perte des zones d'ellipsoïde et d'interdigitation observée dans la TCO, était présente chez tous les patients.

On continue de débattre de la question de savoir si l'ÉIATA est une maladie distincte ou simplement une maladie de l'«AZOOR complex ». L'ÉIATA, le MEWDS et l'AZOOR ont des caractéristiques communes. Les maladies sont plus courantes chez les femmes et se présentent avec des photopsies. La perte de champ visuel observée dans l'AZOOR peut être contiguë ou non à la tache aveugle. Dans le cas de l'AZOOR, il y a généralement une progression de la perte du champ visuel et des anomalies rétiniennes au fil des semaines ou des mois, tandis que la baisse du champ visuel observée dans l'ÉIATA demeure habituellement stable. Les anomalies rétiniennes sont bilatérales dans $39 \%$ des cas d'AZOOR ${ }^{5}$, tandis que l'ÉIATA est généralement unilatéral ${ }^{4,6}$. Parmi les cas que nous avons présentés, tous avaient un scotome unilatéral non progressif, ce qui est plus correspond plus à l'ÉIATA qu'à l'AZOOR.

La disponibilité généralisée de l'autofluorescence est relativement nouvelle, et par conséquent, peu de cas démontrent les anomalies causées par l'ÉIATA. Les zones d'hyperautofluorescence indiquent la détresse de l'EPR tandis que les zones d'hypoautofluorescence indiquent la mort de l'EPR. Par conséquent, cette technologie pourrait être utile pour différencier l'ÉIATA de l'AZOOR. Dans l'AZOOR, il y aura un signal hyperautofluorescent irrégulier dans la zone atteinte qui progressera avec le temps ${ }^{11}$. Lors de l'examen du fond d'œil, il y a une ligne de démarcation autour de la zone atteinte, généralement de couleur orange, qui est mieux visible sur l’imagerie autofluorescente ${ }^{11}$. Cette ligne hyperautofluorescente qui est observée entre la partie normale et la partie atteinte de la rétine est une caractéristique déterminante de l'AZOOR ${ }^{11}$. En plus d'une progression zonale fréquente, Mrejen et ses collègues ${ }^{11}$ ont proposé les critères d'autofluorescence du fond d'œil suivants pour poser un diagnostic d'AZOOR : une étroite ligne de démarcation hyperautofluorescente entre les parties atteintes et saines de la rétine et un modèle trizonal de dégénérescence. Le modèle trizonal peut être vu en autofluorescence comme une zone d'autofluorescence normale en dehors de la région atteinte (zone 1), une zone d'hyperautofluorescence (zone 2) et une zone d'hypoautofluorescence (zone 3). Dans le cas 2, pour lequel une imagerie autofluorescente a été réalisée, on n'a pas observé de ligne hyperautofluorescente démarquant le tissu anormal ni de modèle trizonal. Il y avait beaucoup plus d'hypoautofluorescence papillaire dans l'œil en cause que dans l'œil normal, correspondant à la zone d'atrophie péripapillaire. Le signal était plus clair autour du nerf optique, mais cela semble être égal dans les deux yeux. Chez les patients souffrant d'un AZOOR, le schéma trizonal peut également être observé sur la TCO comme une zone normale à l'extérieur des tissus en cause (zone 1), une zone de dépôts drusénoïdes sousrétiniens (zone 2) et une zone de perte de photorécepteurs (zone 3). Ce modèle n'a été observé chez aucun des patients atteints d'ÉIATA dans le présent rapport. L'absence de modèle trizonal en autofluorescence ou par TCO peut prêter à croire que l'ÉIATA est une condition distincte.

L'ÉIATA a des caractéristiques semblables à celles du MEWDS, et certains soutiennent que les taches blanches caractéristiques dans ce dernier cas ne sont pas visibles se sont résolues dans le cas de l'ÉIATA. Le MEWDS est généralement précédé d'une maladie de type viral et comporte des lésions blanches au niveau des photorécepteurs qui sont visibles pendant quelques semaines (fig. 4A). Généralement, la vision se rétablit complètement, mais après la résolution, le patient développe une granularité de pigment maculaire permanente qui est considérée comme pathognomonique du MEWDS (fig. 4B). Même si ces patients étaient au stade aigu, aucun n'avait de taches blanches rétiniennes et tous ont déclaré n'avoir eu aucun symptôme de type viral. De plus, la granularité maculaire n'était présente dans aucun de nos cas.

Il a été proposé que si le scotome entourant la tache aveugle régresse, cela suggère un diagnostic de MEWDS, alors que l'atrophie péripapillaire associée à la persistance du scotome conduit à un diagnostic de choroïdite multifocale, et que la progression du scotome est indicative d'une $\mathrm{AZOOR}^{10}$. Malheureusement, cette classification ne prend pas en compte d'autres caractéristiques. La choroïdite multifocale est associée à l'uvéite antérieure, ainsi qu'à la présence de multiples lésions choroïdiennes gris-jaune qui deviennent des cicatrices perforées au fil du temps. Malgré l'absence de résolution ou de progression, aucun des trois cas ne présentait de lésions rétiniennes correspondant à ces caractéristiques de la choroïdite multifocale. 


\section{CONCLUSION}

Bien que l'ÉIATA soit mal compris, il est important que les praticiens des soins oculovisuels en connaissent les caractéristiques. D'autres exemples de cette maladie rare peuvent aider à définir et à classer le diagnostic. Nos cas ne correspondent pas à d'autres rétinopathies externes et peuvent indiquer que l'ÉIATA est une maladie distincte de l'AZOOR ou du MEWDS. Un élargissement de la tache aveugle unilatéral non progressif avec des résultats correspondants sur ERGmf sans indications d'autres troubles de la rétine externe est compatible avec un diagnostic d'ÉIATA. En général, le patient présente des symptômes aigus de perte de vision et de photopsie dans le champ temporal. Les symptômes s'atténuent généralement au fil du temps, mais les anomalies du champ visuel et de l'ERGmf demeurent.

AUTEUR-RESSOURCE

Amiee Ho, O.D.

amieeho@pacificu.edu

DIVULGATION DES INTÉRÊTS

soutien financier : aucun

Les auteurs déclarent n'avoir aucun conflit d'intérêts.

\section{RÉFÉRENCES}

1. Fletcher WA, Imes RK, Goodman D, et al. Acute idiopathic blind spot enlargement. A big blind spot syndrome without optic disc edema. Arch Ophthalmol 1988;106:44-9.

2. Jiang L-B, Shen C-Y, Chen F, et al. Clinical features of retinal diseases masquerading as retrobulbar optic neuritis. Chin Med J 2013;126:3301-6.

3. Sugahara M, Shinoda K, Matsumoto SC, et al. Outer retinal microstructure in a case of acute idiopathic blind spot enlargement syndrome. Case Rep Ophthalmol 2011;2:116-22.

4. Volpe NJ, Rizzo JF, Lessell S. Acute idiopathic blind spot enlargement syndrome: a review of 27 new cases. Arch Ophthalmol 2001;119:59-63.

5. Monson DM, Smith JR. Acute zonal occult outer retinopathy. Surv Ophthalmol 2011;56:23-35.

6. Liu X, Chen B, Zhang M, et al. Clinical features and differential diagnosis of acute idiopathic blind spot enlargement syndrome. Eye Sci 2014;29:143-50.
7. Cooper ML, Lesser RL. Prolonged course of bilateral acute idiopathic blind spot enlargement. J Clin Neuroophthalmol 1992;12:173-7.

8. Kondo N, Kondo M, Miyake Y. Acute idiopathic blind spot enlargement syndrome: prolonged retinal dysfunction revealed by multifocal electroretinogram technique. Am J Ophthalmol 2001;132:126-8.

9. Watzke RC, Shults WT. Clinical features and natural history of the acute idiopathic enlarged blind spot syndrome. Ophthalmology 2002;109:1326-35.

10. Trese MGJ, Cohen SR, Besirli CG. Recovery of outer retina in acute idiopathic blind spot enlargement (AIBSE). Am J Ophthalmol Case Rep 2016;1:13-5.

11. Mrejen S, Khan S, Gallego-Pinazo R, et al. Acute zonal occult outer retinopathy: a classification based on multimodal imaging. JAMA

Ophthalmol 2014;132:1089-98. 